\title{
PROGRAMA GRANDES DESAFIOS PARA A ENGENHARIA - GCSP DA ESCOLA DE ENGENHARIA DA UFMG
}

DOI: 10.37702/2175-957X.COBENGE.2021.3707

Carmela Maria Polito Braga - carmela@ufmg.br

UFMG

Rua Bacupã 77

31270-360 - Belo Horizonte - MG

Ana Liddy Cenni de Castro Magalhães - analiddy@gmail.com Universidade Federal de Minas Gerais

Rua Tomé de Souza 1225

30140-131 - Belo Horizonte - MG

DIMAS ALBERTO GAZOLLA - dagap@cce.ufmg.br UNIVERSIDADE FEDERAL DE MINAS GERAIS

Av. Presidente Antônio Carlos 6627

31270-901 - Belo Horizonte - MG

Luiz Machado - luizm@ufmg.br

Universidade Federal de Minas Gerais

Rua Professor Manoel Casassanta 262

31310-590 - Belo Horizonte - MG

Marcelo Cardoso - marcelocardoso@globo.com

Universidade Federal de Minas Gerais

Rua Ludgero Dolabela 249

30441-051 - Belo Horizonte - MG

Maria Teresa Paulino Aguilar - teresa@ufmg.br

UFMG

Rua Bueno Brandão 446

31010-060 - Belo Horizonte - MG

ROBERTO MÁRCIO DA SILVA - ROBERTO@DEES.UFMG.BR

UFMG

RUA ILHA GRANDE 840

31555-030 - BELO HORIZONTE - MG 
Virginia sampaio teixeira ciminelli - virginia.ciminelli@gmail.com

UFMG

R. Passa Tempo 361

30310-760 - Belo Horizonte - MG

Resumo: Apresenta-se a estrutura do Programa Acadêmico Grandes Desafios para a Engenharia (Grand Challenge Schollars Program - GCSP) da Escola de Engenharia da UFMG, vinculado à National Academy of Engineering - NAE. Esse Programa Acadêmico tem uma visão aspiracional de formação de engenheiros preparados para os desafios da Engenharia no século XXI, e é baseado em metodologia integradora, voltada para a formação de competências críticas, com foco em soluções para problemas globais. Ele cria oportunidades para cooperar, bem como desenvolver competência multicultural e multidisciplinar, competência social e comprometimento. Atualmente, os cursos da EEUFMG estão se adaptando para atender às novas diretrizes nacionais para cursos de Engenharia no Brasil, publicadas em janeiro de 2019, e o GCSP pode ser visto como um programa piloto, capaz de transformar a metodologia, a mentalidade e as ferramentas educacionais e tecnológicas que utiliza, em alternativa para todos os cursos de engenharia da UFMG. O Programa iniciou suas atividades na Escola de Engenharia em 2020, definindo critérios para o seu funcionamento, sua organização interna, e iniciando a apresentação do Programa aos estudantes, mesmo com os desafios e limitações impostos pela pandemia de Covid-19. Apresenta-se, também, a primeira iniciativa do Programa em 2020, a Chamada No.01/2020 - Covid-19, de acesso amplo aos estudantes interessados, e as iniciativas propostas para 2021.

Palavras-chave: GCSP, programa acadêmico, grandes desafios para a engenharia, metodologia integradora, formação de competências. 
 \\ PROGRAMA GRANDES DESAFIOS PARA A ENGENHARIA - GCSP DA ESCOLA DE ENGENHARIA DA UFMG}

\section{INTRODUÇÃO}

A Escola de Engenharia da UFMG desenvolve vários projetos e programas acadêmicos que apoiam iniciativas de modernização dos currículos dos cursos de Engenharia. Dentre esses programas, encontra-se o projeto Grand Challenge Scholars Program - GCSP da EEUFMG, vinculado à National Academy of Engineering - NAE, aprovado e iniciado em 2020.

O GCSP objetiva contribuir com a implantação de programas de formação ao redor do mundo, comprometidos com uma formação que desenvolva competências críticas voltadas para a solução de problemas relacionados aos Grandes Desafios para a Engenharia no século XXI. Estes desafios foram relacionados, em 2008, por uma comissão formada por 18 pessoas convidadas, entre engenheiros, tecnólogos e futuristas, como o cofundador do Google, Larry Page, e o futurista Ray Kurzweil. Os 14 desafios apontados abordam quatro temas transversais, quais sejam, sustentabilidade, saúde, segurança e alegria de viver, incorporados ao programa GCSP. Eles apontam questões urgentes para a sociedade moderna e exigem inovação nas soluções de engenharia. Cabe aos cursos de engenharia a responsabilidade de preparar as novas gerações de engenheiros com ferramentas para enfrentar esses desafios e criar soluções sustentáveis, expondo os alunos a esses Grandes Desafios durante seus cursos de graduação. Engenheiros bem preparados podem atuar localmente, mas pensar globalmente, produzindo conhecimento e desenvolvendo soluções que possam ser transferidas, para serem aplicadas em outros locais com problemas semelhantes.

Morell et al. (2008) analisam os desafios da formação de engenheiros preparados para lidar com as questões relevantes em um mundo globalizado e de economias baseadas no conhecimento, que proporcionem melhoria da qualidade, redução de custos, atendimento às necessidades do consumidor e produtos inovadores. Morell (2010) discute o papel e as oportunidades que os professores de engenharia têm para responder aos desafios enfrentados pela educação em engenharia voltada para as questões relevantes do século XXI. Neste trabalho, são descritos o perfil e as características desejadas para o professor ideal de engenharia, segundo uma pesquisa conduzida juntamente com 0 presidente da Plataforma de Estudantes para o Desenvolvimento da Educação em Engenharia (SPEED) em 2009, considerando serem os professores os catalizadores desse processo. O professor ideal, afirma, precisa ser, simultaneamente, engenheiro e professor: ser especialista técnico, comunicar-se de forma eficaz e acompanhar as tendências em sua disciplina, ser professor efetivo e mentor, ser comprometido com a cidadania global e praticar a engenharia. Esse entendimento é consistente com o Programa GCSP e importante para sua implementação, pois este é baseado na mentoria docente dos estudantes, desde o seu ingresso, até a conclusão dos requisitos de formação. Essa mentoria é devida em todas as atividades de cada estudante, que vão desde a escolha das disciplinas a serem cursadas, à escolha do grande desafio e de um projeto a ser desenvolvido, com orientação, mas propiciando a autonomia necessária ao estudante.

A rede NAE/GCSP conta, atualmente, com 71 programas em universidades americanas, 14 em universidades na Europa e Ásia, 1 na Austrália, 2 no Brasil, sendo o da Escola de Engenharia da UFMG o primeiro a ser aprovado, e o da Faculdade de Engenharia Mauá, o segundo. Mas há sempre novas propostas sendo submetidas ao comitê de revisão de propostas da coordenação da rede GCSP e novos programas sendo aprovados em todo 
o mundo. Vários trabalhos publicados discutem a formação de engenheiros baseada nos princípios do Programa NAE/GCSP (MORELL, 2010, KATSOULEAS; MILLER; YORTSOS, 2013) e implementações do Programa GCSP em diferentes universidades (DONARER et al., 2017, Davies, 2020). Neste contexto, Morell et al. (2018) apresentam o programa de formação de lideranças em engenharia para o século XXI, implementado na Universidade de Beihang, que também é baseado em formação de competências, como o GCSP.

Cada universidade deve elaborar uma proposta para implementação do seu Programa GCSP, respeitando as orientações do comitê internacional, em que apresenta uma visão e a estrutura acadêmica da escola/faculdade, os grandes desafios escolhidos como referência para o Programa, a estratégia de implementação dele, considerando-se os recursos, a cultura e as normas internas, formas de seleção de alunos, avaliação, acompanhamento e reconhecimento dos alunos. Os estudantes que completarem os requisitos definidos para o Programa fazem jus ao certificado emitido pela coordenação local e a uma carta de recomendação assinada pelo presidente da NAE.

Destaca-se que cada Programa GCSP deve buscar financiamento, por meio de patrocínio de empresas ou de agências de fomento. O Programa da Escola de Engenharia iniciou, apenas, com recurso remanescente do patrocínio obtido para a realização do GCSP Workshop Brasil, em 2019, que é usado para custeio operacional inicial.

A adesão da Escola da Engenharia ao GCSP ocorreu por meio da construção coletiva da proposta aprovada, por uma equipe constituída de professores dos diversos departamentos da Escola, a convite do vice-diretor. Relacionam-se quatro motivações para a criação desse Programa na EEUFMG:

1. Compromisso de formar engenheiros que sejam tecnicamente preparados, capazes de pensar de forma diferente e capazes de transformar uma ideia em uma solução viável.

2. Uso de metodologia integradora e multidisciplinar, com foco em soluções para problemas globais.

3. Criação da visão aspiracional de uma engenharia preparada para o Século XXI, comprometida com a continuação da vida no planeta, tornando o mundo mais sustentável, seguro, saudável e alegre.

4. Cooperação em rede internacional, o que permite conhecer e debater com colegas de outros países soluções de problemas relevantes para a engenharia, cooperar em oportunidades identificadas em rede e ganhar visibilidade no cenário internacional.

Um Programa GCSP bem-sucedido ajuda a Escola de Engenharia a construir novas abordagens e metodologias para os cursos de engenharia, com uma visão integradora, focada no desenvolvimento de soluções para problemas que são motivo de preocupação em todo o mundo. Este Programa cria uma oportunidade para cooperar, bem como desenvolver competência multicultural e multidisciplinar, competência social e comprometimento. Atualmente, os cursos da EEUFMG estão se adaptando para atender às novas diretrizes nacionais para cursos de engenharia no Brasil, publicadas em janeiro de 2019. A experiência do GCSP pode ser vista como um programa piloto, que busca transformar a metodologia, a mentalidade e as ferramentas educacionais e tecnológicas utilizadas em todos os 11 cursos de engenharia da UFMG.

Apresenta-se na seção 2 o Programa GCSP em implantação na EEUFMG, na seção 3 a organização interna e o funcionamento, na seção 4 as primeiras atividades durante o ano de 2020, o plano de trabalho para o ano de 2021, e na seção 5 as considerações finais do trabalho. 


\section{O PROGRAMA GCSP DA EEUFMG}

O Programa GCSP da EEUFMG oferece um caminho formativo, para conectar com facilidade os alunos do Programa aos recursos disponíveis, e criar seu currículo do GCSP dentro de seus currículos de graduação (uma trajetória curricular no Programa). Ele é constituído por cinco componentes, que visam atender aos princípios básicos de formação das competências críticas:

1) Competência em talentos: pesquisa orientada; experiência criativa no tópico GCSP.

2) Competência multidisciplinar: compreensão da multidisciplinaridade de soluções de sistemas de engenharia - currículo.

3) Competência viável de negócios/empreendedorismo: entendimento do modelo de negócios viável para implementação da solução.

4) Competência multicultural: mentalidade planetária; consideração de questões culturais para garantir a aceitação das soluções de engenharia propostas.

5) Competência de consciência social: entendendo que a Engenharia deve servir às pessoas e à sociedade.

A estrutura flexível de currículos da EEUFMG, definida para os cursos de graduação da UFMG, estabelece que o currículo dos cursos deve oferecer aos alunos a possibilidade de cursar uma Formação Complementar - FC. A FC deve abranger temas relacionados ao curso de graduação, mas em um tópico que represente uma fronteira de conhecimento descoberta pelo currículo original, como mostra a Figura 1. Essa estrutura flexível permite implementar o currículo multidisciplinar do GCSP, conforme exigido pelo Programa. Com onze cursos de engenharia, é possível fornecer muitas opções de disciplinas, incluindo algumas de outras unidades acadêmicas.

Além dos cursos, algumas novas atividades acadêmicas foram propostas para atender aos objetivos do GCSP. Os alunos selecionados para participar do Programa serão instruídos por seus mentores a submeterem seu plano específico de formação complementar GCSP ao Colegiado do seu curso para aprovação. Esta formação complementar no GCSP permitirá que os alunos desenvolvam as habilidades de formação exigidas pelo Programa de maneira aplicável, em um dado projeto, voltado para um dos grandes desafios, preferencialmente em parceria com uma empresa ou comunidade.

Os grandes desafios assumidos para o Programa foram escolhidos considerando-se o entendimento da EEUFMG quanto às demandas prioritárias da cidade de Belo Horizonte, do Estado de Minas Gerais e do Brasil, bem como as competências instaladas na EEUFMG, capazes de contribuir com o desenvolvimento de projetos. São os desafios do GCSP da EEUFMG:

- recuperar e melhorar a infraestrutura urbana;

- prover acesso a água limpa e tratar as águas residuais;

- desenvolver soluções de energia limpa e segura;

- desenvolver mentalidade e soluções sustentáveis;

- desenvolver recursos para aprendizado personalizado avançado.

Considera-se a inserção social dos projetos uma questão fundamental, de forma a ser um exercício permanente de aplicação dos conhecimentos e competências da Engenharia para melhorar as condições de vida das pessoas, sobretudo daquelas que têm necessidades fundamentais ainda não atendidas. 
Figura 1: Estrutura geral dos cursos da Escola de Engenharia da UFMG

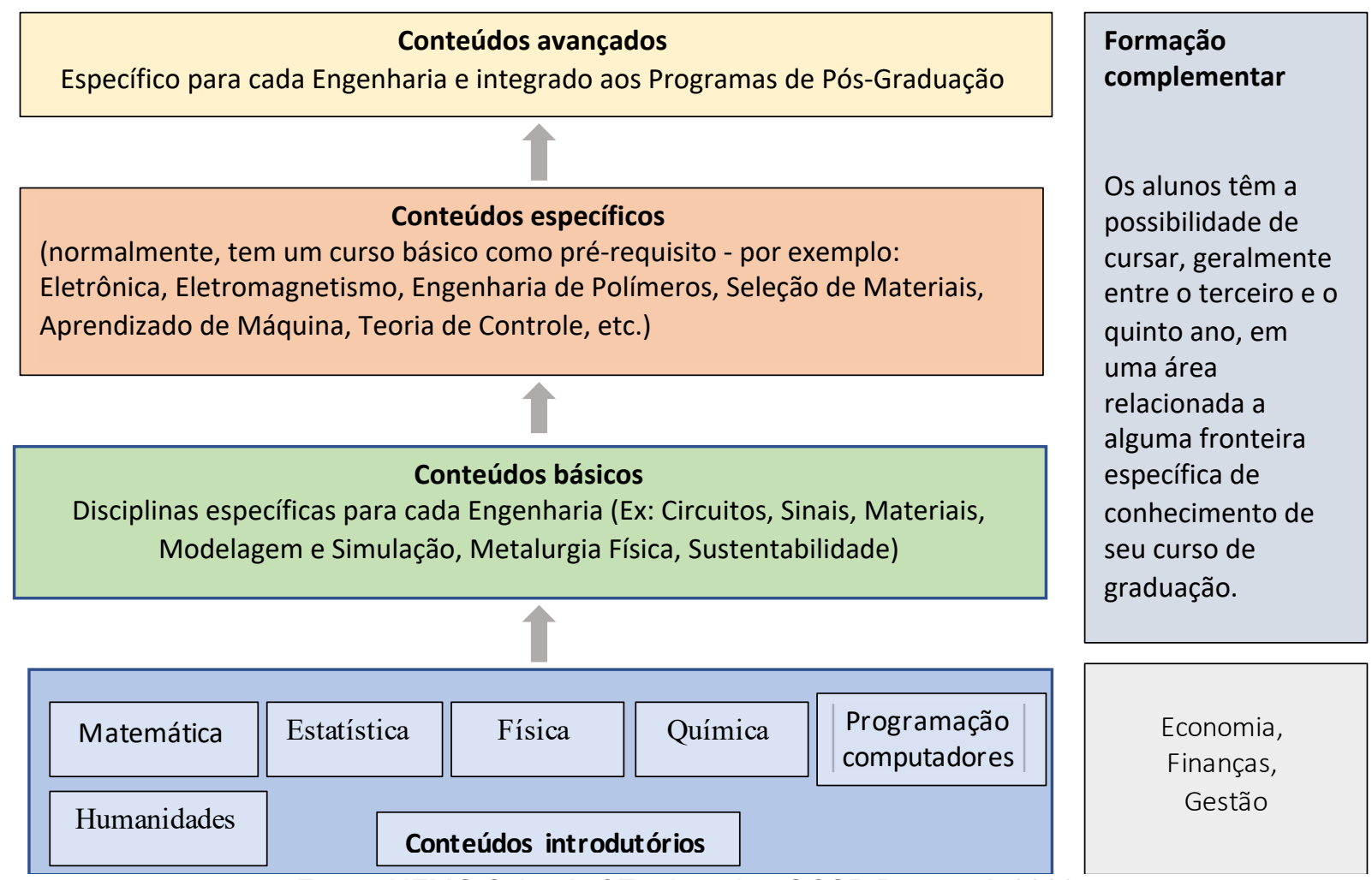

Fonte: UFMG School of Engineering GCSP Proposal, 2020.

Outros recursos importantes incluem as definições normativas da EEUFMG, que estabelecem uma carga horária mínima de atividades acadêmicas complementares a serem realizadas pelos alunos em cada curso. Algumas dessas atividades são consideradas como alternativas entre os componentes do Programa e podem ser integralizadas pelos estudantes nos currículos dos seus cursos e no GCSP.

Uma estratégia fundamental do processo de formação no Programa GCSP é a mentoria docente, que proporciona o acompanhamento integral de cada estudante, durante todo o seu tempo de permanência no Programa. O mentor é o professor responsável por acompanhar e orientar a formação do aluno no Programa, de forma que este vivencie os cinco componentes curriculares baseados nas competências do GCSP. Considera-se que a mentoria de cada estudante no programa GCSP e a orientação do projeto de pesquisa ou desenvolvimento deles podem ser distintas. Em outras palavras, o mentor do estudante no Programa pode ser o orientador do projeto que ele está desenvolvendo, também, mas não necessariamente.

\subsection{Componentes do programa GCSP EEUFMG}

Apresentam-se os componentes do Programa, objetivando o desenvolvimento das cinco competências críticas nos estudantes, mostrando a forma de implementação de cada uma na Escola de Engenharia, a partir dos recursos disponíveis. 
- Competência multidisciplinar (via currículo): caminho formativo a partir da estrutura curricular flexível da UFMG, por meio de um plano de Formação Complementar (240h), submetido ao Colegiado de curso do aluno. Essa carga horária foi escolhida para atender, também, ao requisito mínimo de integralização de uma Formação Complementar, conforme norma interna da UFMG.

Cada aluno deve escolher um dos Grandes Desafios e selecionar as disciplinas da Tabela 1 (mínimo de 180h), além das exigidas ou obrigatórias (60h), inerentemente multidisciplinares e criadas para os objetivos do Programa. As disciplinas sugeridas são relacionadas para cada Grande Desafio de Engenharia deste Programa GCSP, mas outras também podem ser escolhidas, conforme sugerido pelo mentor do aluno, de acordo com cada projeto específico.

O currículo prevê uma atividade, denominada Imersão Acadêmica, que compreende uma combinação de visitas ou estágio em uma empresa ou município. Durante esse período, os alunos e seu mentor identificarão problemas relevantes ou questões técnicas a serem tratadas pelos projetos do GCSP.

Tabela 1: Currículo interdisciplinar

\begin{tabular}{|c|c|}
\hline \multicolumn{2}{|c|}{ Conteúdos básicos para todos os estudantes do GCSP (carga horária mínima de 60h) } \\
\hline $\begin{array}{l}\text { Introdução ao Programa GCSP (Nova) (15h) } \\
\text { (Obrigatória) }\end{array}$ & $\begin{array}{l}\text { Eletrônica, Metrologia e } \quad \text { Fundamentos } \\
\text { Instrumentação (Nova) (30h) (Optativa) }\end{array}$ \\
\hline $\begin{array}{l}\text { Princípios de Sustentabilidade (Nova)(15h) } \\
\text { (Obrigatória) }\end{array}$ & $\begin{array}{l}\text { Inovação e Solução de Problemas (Nova) (15h) } \\
\text { (Obrigatória) }\end{array}$ \\
\hline EMA083 Desenho Mecânico (60h) (Optativa) & Ética em Engenharia (Nova) (15h) (Obrigatória) \\
\hline $\begin{array}{l}\text { Simulação e Modelagem em Engenharia } \\
\text { (Nova) (30h) (Optativa) }\end{array}$ & $\begin{array}{l}\text { EPD064 - Tecnologia e Sociedade (60h) } \\
\text { (Optativa) }\end{array}$ \\
\hline $\begin{array}{l}\text { Internato Curricular Geral Urbano e Rural } \\
\text { (60h) (Optativa) }\end{array}$ & $\begin{array}{l}\text { Fundamentos de Programação Orientada a Objetos } \\
\text { (Nova) (30h) (Optativa) }\end{array}$ \\
\hline \multicolumn{2}{|c|}{ Grande Desafio I: Recuperar e melhorar a infraestrutura urbana } \\
\hline $\begin{array}{l}\text { ETG011 Análise de Sistemas de Transporte } \\
\text { (60h) }\end{array}$ & EHR007 Hidrologia Aplicada (60h) \\
\hline $\begin{array}{l}\text { ETG051 Planejamento de Transportes } \\
\text { Urbanos e Regionais }(60 \mathrm{~h})\end{array}$ & ESA612 Tratamento de Esgotos Sanitários (60h) \\
\hline ETG036 Tráfego Urbano (60h) & ESA009 Controle Ambiental (60h) \\
\hline ETG037 Transportes Públicos (60h) & $\begin{array}{l}\text { ESA014 Tratamento de Águas de Abastecimento } \\
(60 \mathrm{~h})\end{array}$ \\
\hline $\begin{array}{l}\text { EHR018 Engenharia de Recursos Hídricos } \\
(45 \mathrm{~h})\end{array}$ & ESA126 Controle de Poluição Atmosférica (60h) \\
\hline $\begin{array}{l}\text { ENG - Imersão Acadêmica I - Cidades } \\
\text { (Obrigatório) }(60 \mathrm{~h})\end{array}$ & $\begin{array}{l}\text { EPD048 Tópicos em gestão da Produção: Gestão } \\
\text { Ambiental (60h) }\end{array}$ \\
\hline \multicolumn{2}{|c|}{ Grande Desafio II: Fornecer acesso à água, respeitando seus diversos usos, e tratar águas residuais } \\
\hline ENG090 - Reuso da Água (30h) & $\begin{array}{l}\text { Especiação e Reações em Meio Aquoso (nova) } \\
(30 \text { h) }\end{array}$ \\
\hline EHR007 - Hidrologia Aplicada (60h) & EQM056 - Processos Industriais I (60h) \\
\hline $\begin{array}{llll}\text { ESA014 Tratamento de Águas } & \text { de } \\
\text { Abastecimento (60h) } & & & \end{array}$ & $\begin{array}{l}\text { EMN026 - Geologia de Engenharia e Hidrogeologia } \\
\text { Aplicada (60h) }\end{array}$ \\
\hline $\begin{array}{l}\text { EHR018 - Engenharia de Recursos Hídricos } \\
(45 \mathrm{~h})\end{array}$ & $\begin{array}{l}\text { ESA125 - Controle da Poluição do Solo e das Águas } \\
\text { Subterrâneas ( } 60 \mathrm{~h})\end{array}$ \\
\hline $\begin{array}{llll}\text { ESA613 }- \text { Tratamento de } & \text { Efluentes } \\
\text { Industriais }(60 \mathrm{~h})\end{array}$ & $\begin{array}{l}\text { ESA612 Tratamento de Esgotos Sanitários } \\
(60 \mathrm{~h})\end{array}$ \\
\hline
\end{tabular}




\begin{tabular}{|c|c|}
\hline $\begin{array}{l}\text { ENG - Imersão Acadêmica II - Água } \\
\text { (Obrigatório) (60h) }\end{array}$ & \\
\hline \multicolumn{2}{|c|}{ Grande Desafio III: Desenvolver soluções energéticas limpas e seguras } \\
\hline $\begin{array}{llll}\text { EEE024 - Fundamentos de } & \text { Energias } \\
\text { Alternativas }(30 \mathrm{~h}) & & \\
\end{array}$ & $\begin{array}{l}\text { ENG033 - Tópicos em Engenharia Mecânica C: } \\
\text { Tecnologia solar - Fundamentos e aplicações }(60 \mathrm{~h})\end{array}$ \\
\hline ELE045 - Geração de Energia Elétrica (60h) & ENU002 - Questões Energéticas (60h) \\
\hline EHR020 - Usinas Hidrelétricas (60h) & $\begin{array}{l}\text { ENG091 - Recuperação de Produtos e Energia do } \\
\text { Saneamento Ambiental (60h) }\end{array}$ \\
\hline $\begin{array}{l}\text { EEE934 - Armazenadores de } \text { Energia } \\
\text { Elétrica (PPGEE) (60h) }\end{array}$ & $\begin{array}{l}\text { Materiais para Geração e Acúmulo de Energia (30h) } \\
\text { (nova) }\end{array}$ \\
\hline \multicolumn{2}{|l|}{$\begin{array}{l}\text { ENG - Imersão Acadêmica III - Energia - } \\
\text { (Obrigatório) (60h) }\end{array}$} \\
\hline \multicolumn{2}{|c|}{ Grande Desafio IV: Desenvolver mentalidades e soluções sustentáveis } \\
\hline EMT074 - Ciência dos Materiais (60h) & $\begin{array}{l}\text { ENG090 - Mudança do Clima e Mercado de Carbono } \\
(30 \mathrm{~h})\end{array}$ \\
\hline ENG033 - Biocombustíveis Líquidos (45h) & $\begin{array}{l}\text { EMT094 Seleção de Materiais e Processos (nova) } \\
(30 \mathrm{~h})\end{array}$ \\
\hline $\begin{array}{l}\text { Processos Químicos voltados para } \\
\text { sustentabilidade (nova) (45h) }\end{array}$ & $\begin{array}{l}\text { Processos Aplicados ao tratamento de Resíduos } \\
\text { Sólidos e Efluentes Aquosos (para recuperação de } \\
\text { materiais) (nova) (30h) }\end{array}$ \\
\hline $\begin{array}{l}\text { Recuperação de Áreas Degradadas e Usos } \\
\text { Futuros (nova) (30h) }\end{array}$ & ELT071 Automação Residencial (30h) \\
\hline \begin{tabular}{llll|} 
ENG $\quad$ Imersão Acadêmica IV & - \\
Sustentabilidade (Obrigatório) $(60 \mathrm{~h})$ & &
\end{tabular} & $\begin{array}{l}\text { EMN017- Aproveitamento Sustentável de Recursos } \\
\text { Minerais (60h) }\end{array}$ \\
\hline \multicolumn{2}{|c|}{ Grande Desafio V: Desenvolver recursos para o aprendizado personalizado avançado } \\
\hline $\begin{array}{l}\text { DIP FAE988 - Didática e Docência: O Ensino } \\
\text { como Objeto de Estudo ( } 30 \mathrm{~h})\end{array}$ & Disciplina de Engenharia na área desejada \\
\hline DIP FAE956-Tecnologias Educacionais (30h) & Disciplina de Engenharia na área desejada \\
\hline MTE 025 - Didática (60h) & Disciplina de Engenharia na área desejada \\
\hline $\begin{array}{l}\text { ENG - Imersão Acadêmica } \quad \text { V } \\
\text { Aprendizagem (Obrigatório) }(60 \mathrm{~h})\end{array}$ & \\
\hline
\end{tabular}

Fonte: UFMG School of Engineering GCSP Proposal, 2020.

- Competência em talento: todos os alunos selecionados para o Programa devem participar de uma Atividade de Imersão Acadêmica, relacionada ao Grande Desafio escolhido por eles, conforme indicado na Tabela 1. Essa atividade inclui visitas com imersão, por um período, em uma determinada comunidade, cidade, empresa, escola etc., onde seja possível identificar um determinado problema ou demanda a ser resolvida. Os alunos serão convidados a formar grupos multidisciplinares para participar dessa imersão, sendo sempre orientados por pelo menos um professor / mentor do Programa GCSP.

O objetivo é aumentar a conscientização, dar a eles a oportunidade de identificar um problema, discuti-lo com a comunidade ou o grupo, obter aceitação e confiança do grupo para propor uma pesquisa ou desenvolvimento específico, na tentativa de alcançar um resultado positivo para a comunidade, mesmo que seja apenas um benefício parcial.

O projeto de pesquisa e/ou desenvolvimento que cada aluno proporá deverá ser escolhido a partir dessa imersão, tentando compartilhar o problema identificado e as 
responsabilidades envolvidas, com base nas competências específicas de cada aluno, trabalhando em colaboração para alcançar uma solução viável.

- Competência em empreendedorismo: promover a capacidade e a competência no processo de tradução de ideias, invenção e inovação em uma solução viável. Para entender e aplicar o entendimento da economia local e global em projetos, cada estudante do Programa deve escolher, pelo menos uma, entre as atividades relacionadas a empreendedorismo:

- Participar da Oficina de Projetos, Empreendedorismo e Inovação;

- Cursar Sistema de Desenvolvimento do Produto.

- Cursar Projeto de Processos.

- Cursar Inovação para Sustentabilidade.

- Cursar Tópicos Avançados em Inovação.

- Participar no conselho administrativo de uma empresa júnior por um semestre;

- Participar em uma competição nacional ou internacional.

- Competência multicultural: desenvolver e ampliar a conscientização global e a experiência multicultural. Escolher, pelo menos uma, entre as experiências:

- Estudar no exterior por meio de algum programa internacional.

- Realizar estágio no exterior com foco global.

- Participar de projetos focados em um programa global, com alguma experiência com estrangeiros, mesmo que em uma reunião virtual. O EWB - Engineers Without Borders pode ajudar com alguma oportunidade para isso.

- Participar de algum curso de férias, mesmo na UFMG, com a participação de estudantes estrangeiros que ingressam em nossa universidade.

- Participar de algum evento internacional do GCSP, apresentando experiência pessoal no Programa.

Para a opção escolhida, os estudantes do Programa devem justificar como o programa de estudo (Formação Complementar) / estágio / projeto / experiência proposto: a) cultivará a conscientização global, b) promoverá a sensibilidade a múltiplas perspectivas, c) melhorará as habilidades de comunicação em uma língua estrangeira e d) facilitará o desenvolvimento da comunidade.

- Competência em Consciência Social: promover e aprofundar a consciência e a motivação social, a fim de que o profissional traga seus conhecimentos técnicos para desenvolver soluções que sirvam para melhorar a vida de todas as pessoas, principalmente daquelas que mais precisam. Escolher, pelo menos uma, entre as experiências:

- Internato Acadêmico da Engenharia;

- Programa de Internato Curricular;

- Participar do CIPMOI como instrutor de algum curso;

- Participar da iniciativa de Engenharia Solidária;

- Participar de algum projeto do Engenheiros sem Fronteiras - ESF;

- Participar do Cursinho Equalizar como instrutor.

\subsection{Avaliação, Acompanhamento e Reconhecimento}

Os estudantes se reunirão pelo menos duas vezes por período com seu mentor do GCSP. O mentor documentará seu progresso e realizações por meio de uma versão online de seu portfólio GCSP. Uma folha de acompanhamento estará disponível para ajudar os mentores e os alunos. Cada estudante publicará seu trabalho em seu portfólio, para revisão pelo seu mentor do corpo docente, que estará acessível ao Comitê Diretor do GCSP

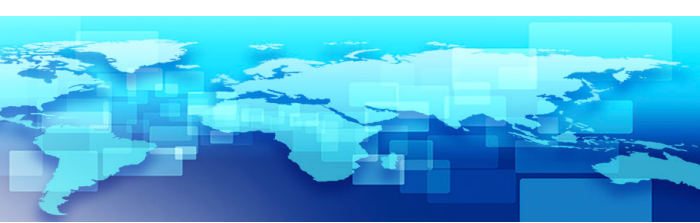


para revisão periódica, duas vezes por ano. Além disso, o Comitê Diretor do GCSP se reunirá periodicamente com os mentores do corpo docente para revisar o progresso e garantir que todos os estudantes continuem a atender aos requisitos do Programa

As avaliações finais do programa individual do aluno serão conduzidas pelo mentor do corpo docente e pelo Comitê Diretor.

Após cada ano do Programa, o Comitê Diretor organizará uma apresentação em pôster para mostrar o trabalho dos alunos, reconhecendo suas contribuições para as questões importantes apresentadas por esses desafios.

Uma cerimônia será conduzida pelo Comitê Diretor, o diretor e o vice-diretor da Escola de Engenharia para conceder o certificado de participação do GCSP aos alunos que concluírem, satisfatoriamente, os cinco componentes curriculares do Programa e premiar os melhores projetos.

\section{ORGANIZAÇÃO INTERNA DO PROGRAMA}

O Programa GCSP da EEUFMG possui um conselho diretor, responsável pela supervisão do mesmo, e um grupo de professores mentores, responsável pelo acompanhamento sistemático dos alunos admitidos no programa.

O conselho diretor é composto pelo vice-diretor da EEUFMG, a(o) coordenador(a) do Programa e professores seniores das diversas áreas de engenharia. Espera-se que este conduza e inove dinamicamente o currículo, mantendo-o atualizado e alinhado às recomendações do NAE e ao perfil e estrutura curricular da EEUFMG, com foco na natureza multidisciplinar do Programa. Ele é responsável por receber os alunos admitidos, orientálos em relação ao currículo e habilidades, e ajudá-los a encontrar um mentor e um tema para o projeto de pesquisa, que deve estar alinhado com os grandes desafios definidos acima. Além disso, deve desenvolver um Guia de Referência do Programa para mentores (do Programa) e orientadores (de projetos), definindo papéis e diretrizes que garantam afinidade com os objetivos do Programa.

A avaliação e os relatórios produzidos pelos mentores para cada bolsista serão compilados pelo(a) coordenador(a) do conselho diretor, permitindo que este(a) produza um relatório geral do Programa a cada semestre e ao final de cada ano. Além disso, o(a) coordenador(a), com o apoio do conselho, deve interagir com o(a) diretor(a) da Diretoria de Relações Internacionais - DRI, buscando alternativas concretas para a participação dos estudantes em programas internacionais, além de estabelecer parcerias com instituições e empresas que possam ser financeiramente benéficas para a iniciativa.

\section{ATIVIDADES DO PROGRAMA NO PRIMEIRO ANO}

O Programa da EEUFMG foi aprovado pela NAE/GCSP em abril de 2020 e, um mês após a aprovação, iniciou seu processo de organização interna, discussões conceituais e procedurais para a implementação do Programa na Escola de Engenharia. Optou-se por iniciar a divulgação e a atuação do Programa junto aos alunos com uma chamada ampla de trabalhos, motivada pelos desafios apresentados pela pandemia causada pelo coronavírus a várias áreas do conhecimento, bem como de viver em meio ao conhecimento ainda em desenvolvimento e condições incertas.

A chamada GCSP EEUFMG N01/2020_Covid-19, do Programa GCSP/EEUFMG, era voltada aos alunos de todos os cursos de graduação da Escola de Engenharia, e definia que as propostas deviam contribuir, por meio de abordagem sistêmica, com o uso das metodologias e tecnologias da Engenharia, além de uma mentalidade inovadora, para combater o contágio e os impactos decorrentes da pandemia mundial do novo coronavírus. 
As propostas deviam envolver equipes multidisciplinares, de 3 a 5 membros, com alunos de pelo menos dois cursos de Engenharia. Dado que o trabalho da Engenharia nesse contexto exige cooperação com outras áreas de conhecimento, a participação de estudantes de outros cursos, externos à Escola de Engenharia, foi considerada bem-vinda. O processo de seleção se deu em duas fases: a $1^{\text {a }}$ Fase, de submissão de proposta preliminar, em que a equipe e a proposta poderiam ainda não estar completas, e a $2^{\mathrm{a}}$ Fase, de refinamento da proposta, com mentoria dos professores do grupo GCSP, auxiliando as equipes a melhorarem a proposta, a torná-la mais factível, ajudando a encontrar outros membros para a equipe, se necessário. A seleção final das propostas ocorreu após a revisão destas, por parte das equipes proponentes, considerando as orientações da mentoria. As propostas deveriam escolher uma categoria de interesse entre os grandes grupos de desafios para a Engenharia, mais adaptados e exemplificados para o propósito da chamada (desafios da pandemia coronavirus-19), para familiarizar os estudantes com os desafios:

I. Sustentabilidade e recuperação (infraestrutura em geral - sanitária, habitacional, transportes, recuperação socioeconômica, materiais reutilizáveis, cuidados no descarte de materiais etc.)

II. Segurança (tecnologia e logística para distribuição de recursos, informações equitativas, inteligência artificial, proteção pessoal, coletiva e de profissionais de saúde etc.)

III. Saúde (tecnologias de sanitização/ desinfecção, saneamento urbano e rural, produção de suprimentos/ utensílios, aplicativos para monitorar a saúde, ferramentas para idosos etc.)

IV. Bem-estar social / alegria de viver, com adequação de ambientes e práticas saudáveis de auto isolamento, mitigação de danos sociais etc.

Foi realizado um evento virtual para a apresentação do Programa e lançamento da chamada, com a participação de toda a equipe GCSP, conselho diretor e mentores. Foram recebidas cinco propostas na primeira fase. Após a mentoria na segunda fase, apenas dois grupos enviaram as propostas revisadas e, para estes, foram atribuídos professores mentores, responsáveis pelo acompanhamento e orientação das equipes. Um desses grupos desistiu do projeto após dois meses e o outro grupo se mantém ativo, com projeto desenvolvido, em fase de prototipação. Destaca-se que o período de envio de propostas foi coincidente com a retomada do $1^{\circ}$ semestre letivo de 2020 na UFMG, o que gerou muita ansiedade entre os estudantes, diante das incertezas do novo modo de oferta das disciplinas. De fato, toda a oferta em modo remoto tem sido desafiante para docentes, que precisaram adaptar seus materiais, estratégias, aulas e trabalhos, e para os estudantes, que procuram manter sua motivação, mesmo com a sensação de alguma perda em relação às atividades presenciais em laboratório.

O conselho diretor decidiu adiar o processo de seleção de estudantes para a primeira admissão ao Programa, uma vez que devido a pandemia do COVID-19 a realização de atividades presenciais, como as imersões acadêmicas, estratégicas na proposta metodológica, não podem ser realizadas a contento. Novas avaliações serão feitas ao longo do ano, para definir data e todo processo de seleção, sendo a expectativa de ingresso da primeira turma para 2022/1, mas promoverá atividades de caráter amplo durante os dois semestres de 2021. Serão ofertadas oficinas em temas como responsabilidade social e criatividade, sustentabilidade e plataformas digitais, sendo que o convite para oferta será estendido a todos os docentes, e não somente os membros da equipe do Programa. 0 modo de oferta poderá ser remoto, presencial ou híbrido, conforme proposta do docente e possibilidade de acesso aos laboratórios e outros espaços da Escola de Engenharia. Além disso, serão organizadas mesas redondas e palestras, em modo remoto, para discutir 
alguns dos grandes desafios, em especial aqueles assumidos pelo Programa, expondo todos os alunos da Escola a estes temas.

\section{CONSIDERAÇÕES FINAIS}

A mobilização da equipe, que viria a constituir o conselho diretor e o grupo de mentores, desde as discussões que precederam a elaboração da proposta, contribuiu de forma significativa para a construção coletiva de uma proposta robusta, aproveitando da melhor maneira os recursos disponíveis na Escola de Engenharia e na UFMG. A estrutura curricular flexível e o conceito de formação complementar viabilizaram implementar um currículo que contempla tanto os requisitos do GCSP quanto os dos cursos de engenharia, permitindo aos estudantes cumprir os requisitos do Programa e integralizar os créditos realizados em seu curso, ao mesmo tempo. O envolvimento da equipe desde a elaboração da proposta tornou-a comprometida com ela, participando e contribuindo com as discussões e atividades do Programa.

A conjuntura mundial no ano de aprovação do Programa impôs adiamento na sua plena implementação, mas trouxe desafios e motivação para atividades diversas, de caráter amplo para todos os estudantes, como a primeira chamada de trabalhos - Covid-19. Isso permitiu iniciar o processo de apresentação da equipe e do Programa aos estudantes, mobilizou a equipe para discussões importantes, como o processo de mentoria, e a organização de outras atividades de caráter amplo, como as oficinas e palestras, voltadas para os grandes desafios. Essas iniciativas permitem que a equipe do programa contribua com a inclusão de todos os alunos da Escola de Engenharia, interessados, na discussão dos grandes desafios da engenharia.

\section{Agradecimentos}

Os autores agradecem ao NAE/GCSP, à Diretoria da Escola de Engenharia, que acreditando no valor do Programa, criou a equipe GCSP a convite, e aos mentores do programa, que participaram ativamente da discussão da proposta.

\section{REFERÊNCIAS}

MORELL, L., BORRI, C., HOYER, H. J., RAJALA, S. A., RAMAKRISHNA, S., FOURGER, X., LAPORTE, B., QUADRADO, J. C., PETRIE, M. M. A., FRASER, D. M. IFEES: Enhancing engineering education at a global scale. Revista de Ensino de Engenharia, v. 27, n. 3, p. 5-21, EDIÇÃO ESPECIAL 2008.

MORELL, L. Engineering Education in the 21st Century: Roles, Opportunities and Challenges. International Journal of Technology and Engineering Education. v. 7, n. 2, p. $1-10,2010$

KATSOULEAS, T., MILLER, R., YORTSOS, Y. The NAE Grand Challenge Scholars Program. The Bridge. Summer issue on Undergraduate Engineering Education. NAE. $V$. 43, n. 2, p. 53-56, 2013.

DONAHER, S., DANCZ, C.L. A., PUMBLEE II, J. M., GORDON, A. S., PATEL, K. Reviewing the Current State of Grand Challenge Scholars Programs Across the United States. IN: 2017 ASEE Annual Conference \& Exposition, 2017, Columbus, Ohio. Disponível em: https://www.asee.org/public/conferences/78/papers/18476/view. Acesso em 06 abr. 2021. 
MORELL, L., ZHAO, H., LI, T., WEN, D., RAMIREZ, W. J., SHAN, G. Developing Engineering Leaders for $21^{\text {st }}$ Century. IN: VIII World Engineering Education Forum (WEEF) and X Global Engineering Deans Council, 2018, Albuquerque, New Mexico. Disponível em: https://www.researchgate.net/publication/330792112 Developing Engineering Leaders f or the 21 st Century. Acesso em 07 abr. 2021.

DAVIES, E. Incorporating Grand Challenges and Developing a Grand Challenges Scholars Program at a Large Land Grant University. IN: ASEE Southeastern Annual Section Conference, 2020, Alburn, Alabama. Disponível em: https://sites.asee.org/se/wpcontent/uploads/sites/56/2021/01/2020ASEESE76.pdf . Acesso em 06 abr. 2021.

\section{NAE Grand Challenges Scholars Program.}

http://engineeringchallenges.org/GrandChallengeScholarsProgram.aspx. Acesso em: 27 abr. 2021.

\section{SQUATRIGLIA, C. And the 14 Grand Engineering Challenges of the 21st Century}

Are... Disponível em: https://www.wired.com/2008/02/and-the-14-big/ . Acesso em: 27 abr. 2021.

UFMG School of Engineering GCSP Proposal. Disponível em: http://www.engineeringchallenges.org/File.aspx?id=33929\&v=4941ed96. Acesso em 06 mai. 2021.

\section{GRAND CHALLENGES FOR ENGINEERING PROGRAM - GCSP OF UFMG SCHOOL OF ENGINEERING}

Abstract: The structure of the Grand Challenges Scholars Program - GCSP of the UFMG School of Engineering, linked to the National Academy of Engineering - NAE, is presented. This Academic Program has an aspirational vision of training engineers prepared for the challenges of engineering in the 21st century and is based on an integrative methodology and on the formation of critical skills, with a focus on solutions to global problems. It creates opportunities to cooperate, as well as to develop multicultural and multidisciplinary competence, social competence, and commitment. Currently, undergraduate courses of the UFMG School of Engineering are adapting to meet the new national guidelines for Engineering courses in Brazil, published in January 2019, and the GCSP can be seen as a pilot program, capable of transforming the methodology, the mindset and the educational and technological tools it uses, as an alternative for all engineering courses at UFMG. The Program starts its activities at the School of Engineering in 2020, defining the criteria for its functioning, its internal organization, and starts a presentation of the Program to students, even with the challenges and limitations caused by the Covid-19 pandemic. Also presented is the first initiative of the Program in 2020, Call No.01 / 2020 - Covid-19, with wide access to interested students, and the proposed activities for 2021.

Keywords: GCSP, academic program, grand challenges for engineering, integrative methodology, skills training. 